\title{
Interaction between neoclassical effects and ion temperature gradient turbulence in gradient- and flux-driven gyrokinetic simulations
}

\author{
M. Oberparleiter, ${ }^{1,2, a)}$ F. Jenko, ${ }^{3}$ D. Told, ${ }^{3}$ H. Doerk, ${ }^{2}$ and T. Görler ${ }^{2}$ \\ ${ }^{1}$ Department of Earth and Space Sciences, Chalmers University of Technology, SE-412 96 Gothenburg, \\ Sweden \\ ${ }^{2}$ Max Planck Institute for Plasma Physics, Boltzmannstr. 2, 85748 Garching, Germany \\ ${ }^{3}$ Department of Physics and Astronomy, University of California, Los Angeles, California 90095, USA
}

(Received 7 December 2015; accepted 1 April 2016; published online 21 April 2016)

\begin{abstract}
Neoclassical and turbulent transport in tokamaks has been studied extensively over the past decades, but their possible interaction remains largely an open question. The two are only truly independent if the length scales governing each of them are sufficiently separate, i.e., if the ratio $\rho_{*}$ between ion gyroradius and the pressure gradient scale length is small. This is not the case in particularly interesting regions such as transport barriers. Global simulations of a collisional ion-temperature-gradient-driven microturbulence performed with the nonlinear global gyrokinetic code GENE are presented. In particular, comparisons are made between systems with and without neoclassical effects. In fixed-gradient simulations, the modified radial electric field is shown to alter the zonal flow pattern such that a significant increase in turbulent transport is observed for $\rho_{*} \geqslant 1 / 300$. Furthermore, the dependency of the flux on the collisionality changes. In simulations with fixed power input, we find that the presence of neoclassical effects decreases the frequency and amplitude of intermittent turbulent transport bursts (avalanches) and thus plays an important role for the self-organisation behaviour.

[http://dx.doi.org/10.1063/1.4947200]
\end{abstract}

\section{INTRODUCTION}

The modelling and prediction of cross-field transport of particles, heat, and momentum in magnetically confined plasmas remains one of the central tasks for the development of future fusion power plants. It is a wellestablished fact that the dominant transport channel of the main ion species in the core region of tokamak devices is ion-temperature-gradient driven (ITG) turbulence. ${ }^{1,2}$ Collisions in connection with the toroidal geometry of the magnetic guide field, however, provide another relevant channel: neoclassical transport. ${ }^{3,4}$ In contrast to turbulent mechanisms, it does not possess a critical threshold for the driving pressure gradient and also provides a minimal flux level in transport barriers where turbulence is suppressed. ${ }^{5}$

Often turbulence and neoclassical transport are treated separately in numerical modelling. This is justified by the fact that in the local (flux-tube) limit both effects are formally independent due to the separation of their characteristic length scales. In global simulations, which take the radial profiles of magnetic field geometry, density, and temperature into account, however, the question arises to which degree this decoupling still occurs. This is of special interest for physical situations where global effects are known to be important such as small devices and the previously mentioned transport barriers. A previous study ${ }^{6}$ of the role of collisional effects in global turbulence simulations with fixed gradient profiles found that the total heat conductivity of a collisional

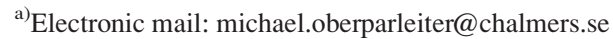

simulation is larger than the sum of the purely neoclassical conductivity for that collisionality and the turbulent conductivity of an otherwise identical non-collisional system. A possible explanation for this can, however, be found without involving neoclassical effects: Collisions themselves damp zonal flows and hence can increase the level of turbulent transport. ${ }^{7,8}$

In this work, we use the global gyrokinetic code GENE $^{9,10}$ to compare collisional simulations which either include or neglect neoclassical effects. In Section II, systems with a fixed background density and temperature gradients are covered where we especially study the role of the scaling parameter $\rho_{*}=\rho_{i} / a$, i.e., the ratio of ion gyroradius and device minor radius as it determines the interaction strength between neoclassical and turbulent phenomena. The modification of the zonal flow patterns by the additional radial electric field is investigated in this context. Additionally, we present how the presence of neoclassical effects affects the dependency on the ion-ion collisionality $\nu_{*}=\nu_{i i} / \omega_{b} \epsilon$, which relates the bounce frequency $\omega_{b}$ of banana orbits to the effective ion-ion collision frequency $\nu_{i i} / \epsilon$ for scattering particles from these orbits.

In Section III, results from simulations with a fixed power input are presented. In particular, it is shown how the presence of neoclassical effects changes the intermittency of turbulence: Due to the additional transport channel without a critical gradient turbulent burst tend to become less frequent. When the power input is varied, it is also demonstrated that the temperature gradient is stiff while heat transport scales with the input power. In the final section, conclusions are drawn from these results. 


\section{EFFECT OF NEOCLASSICAL PHYSICS IN FIXED-GRADIENT SIMULATIONS}

\section{A. Simulation setup}

When the gyrokinetic equations are derived in the $\delta f$ formulation, i.e., the distribution function $f$ is split into a Maxwellian background $F_{0}$ and a small perturbation $\delta f=f_{1}$, they can be formally written as

$$
\mathcal{L} f_{1}+\mathcal{N}\left[f_{1}\right]+\mathcal{B}=C\left(f_{1}\right),
$$

where $\mathcal{L}$ is a linear and $\mathcal{N}$ a nonlinear operator, $C\left(f_{1}\right)$ is the collision operator, and $\mathcal{B}=\boldsymbol{v}_{d} \cdot \nabla F_{0}$ is a term which only depends on the Maxwellian background distribution function $F_{0}$ and the drift velocity $\boldsymbol{v}_{d}$ due to the background magnetic field. This term $\mathcal{B}$ is responsible for the presence of neoclassical effects in the system and consequently called the neoclassical source or drive. In the flux-tube limit, this term only contributes to the toroidally and radially symmetric mode $\left(k_{x}, k_{y}\right)=(0,0)$, which does not couple to the turbulent modes with finite wave numbers leading to the previously mentioned decoupling of neoclassical and turbulent physics. As this is not the case in global simulations, it is straightforward to perform simulations including or excluding neoclassical phenomena with a numerical code based on this equation such as GeNE.

In the simulations presented in this work, circular concentric flux surfaces ${ }^{11}$ are employed where the safety factor profile

$$
q(x / a)=0.854+2.239(x / a)^{2}+0.147(x / a)^{4}
$$

is chosen so that the local values of $q$ and the magnetic shear $\hat{s}$ at the reference position $x=0.5 a$ (half minor radius) are close to the cyclone base case. ${ }^{12}$ This also applies to the inverse aspect ratio $\epsilon(x=0.5 a)=0.18$.

For gradient-driven simulations, we set up the logarithmic gradient profiles of ion temperature and density in either the "peaked" form

$$
\frac{\mathrm{d} \ln T}{\mathrm{~d} \hat{x}}=\kappa_{T} \frac{\cosh \left(\frac{\hat{x}-c_{T}}{w_{T}}\right)^{-2}-\cosh \left(\frac{c_{T}}{w_{T}}\right)^{-2}}{1-\cosh \left(\frac{c_{T}}{w_{T}}\right)^{-2}}
$$

or the "flat-top" form

$$
\frac{\mathrm{d} \ln T}{\mathrm{~d} \hat{x}}=\frac{\kappa_{T}}{2}\left(\tanh \left(\frac{\hat{x}-c_{T}+\delta_{T}}{w_{T}}\right)-\tanh \left(\frac{\hat{x}-c_{T}-\delta_{T}}{w_{T}}\right)\right)
$$

and maintain this initial state in a time-averaged sense by using an adaptive Krook type heating. The radial coordinate $\hat{x}=x / a$ in Eqs. (3) and (4) is normalised to the minor radius $a$. The ion density $n$ and temperature $T$ are normalised to their values $n_{\text {ref }}, T_{\text {ref }}$ at $x=0.5 a$. Time in the simulation is measured in units of $a / c_{s}$ where $c_{s}=\sqrt{T_{\text {ref }} / m_{i}}$ is the ion thermal speed at $x=0.5 a$. The electrons are treated in the adiabatic limit.

We study both types of profiles with the shape and position parameters given in Table I. Their initial state and their
TABLE I. Profile parameters for gradient driven simulations. For their definition see Eqs. (3) and (4).

\begin{tabular}{lcccccc}
\hline \hline Shape & $c_{n, T}$ & $w_{n}$ & $w_{T}$ & $\delta_{n, T}$ & $\kappa_{n}$ & $\kappa_{T}$ \\
\hline Peaked & 0.5 & 0.15 & 0.25 & - & 0.789 & 3.49 \\
Flat-top & 0.5 & 0.05 & 0.05 & 0.25 & 0.789 & 3.49 \\
\hline \hline
\end{tabular}

time average over several hundred time units at around $t=2000 a / c_{s}$ in a typical simulation are shown in Fig. 1 . While some deviation from the initial state can be found, it is not problematic and based on a trade-off with the modification of the system caused by a stronger Krook source.

The gyrokinetic equations are solved on a fixed grid where in configuration space the radial coordinate $x$ and the direction along the field lines $z$ are considered in real space. The field line label $y$, however, is represented in Fourier space. The typical numerical grid is chosen as $\left(N_{x}, N_{k_{y}}, N_{z}, N_{v_{\|}}, N_{\mu}\right)$ $=\left(N_{x}, 32,32,64,32\right)$, where $N_{x}$ is adapted when the system size parameter $\rho_{*}$ is changed to ensure 1-1.33 grid points per ion gyroradius. The y domain is chosen so that every simulation covers $1 / 3$ of the torus. The collisionality is set at the reference position as $\nu_{*}(x=0.5 a)=0.29$ and varies with the geometry and pressure profiles. The plasma is in the banana regime over the entire radial domain, though. The simulation time is chosen to cover at least 2 ion-ion collision times at all radial positions in order to ensure sufficiently converged neoclassical fluxes.

The uncertainties presented in the figures of this article are standard error of the mean estimates based on batch means. The individual batches are formed by grouping the simulation data within a time window of 5 autocorrelation times.

\section{B. System size scaling}

The physical parameter of primary interest is the normalized gyroradius $\rho_{*}=\rho_{i} / a$. Any observed interaction between the ITG turbulence and the neoclassical effects should weaken and disappear when $\rho_{*}$ decreases since the simulations then approach the local (flux tube) limit. The $\rho_{*}$ given here is its value at the position $x / a=0.5$. The local ion gyroradius varies with the temperature and the magnetic field strength. It should also be noted that since both profiles are quite narrow the scaling of the turbulent flows does rather

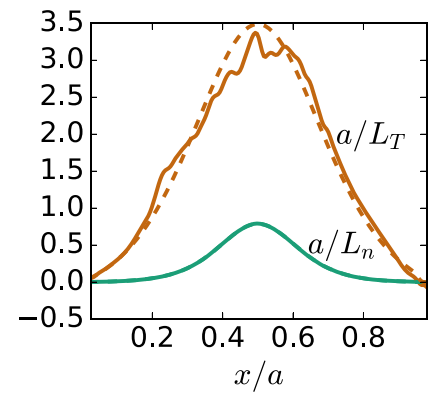

(a) peaked

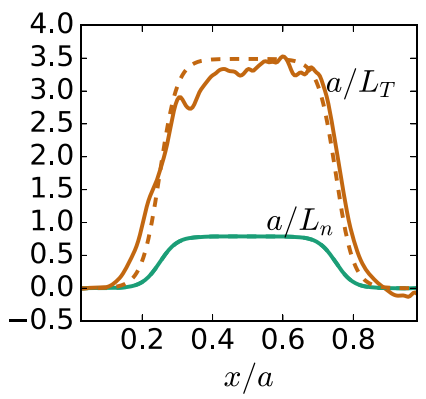

(b) flat-top
FIG. 1. Radial profiles for the logarithmic gradients of $T_{i}$ and $n_{i}$. Dashed: initial state, solid: time average over $500 a / c_{s}$ at $t \sim 2000 a / c_{s}$. 


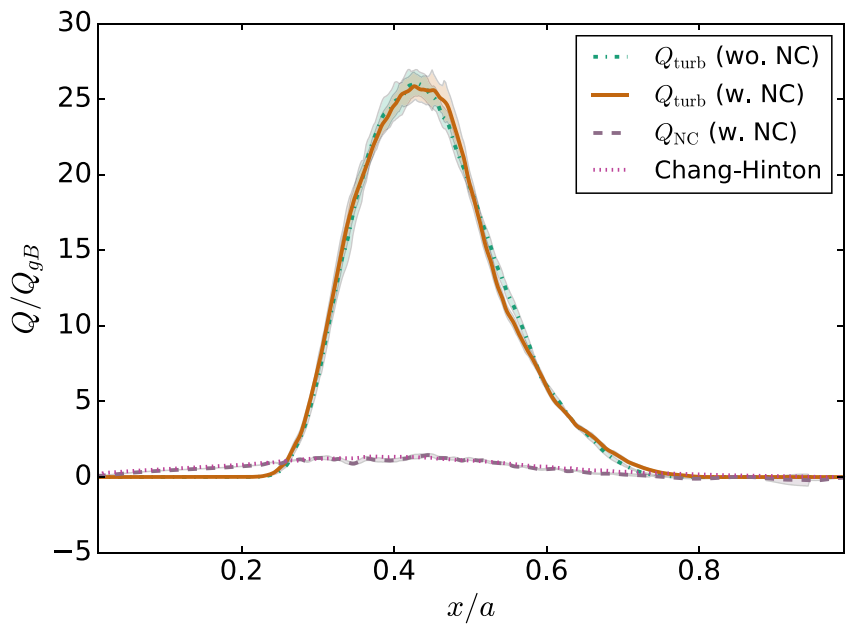

FIG. 2. Neoclassical and turbulent radial heat flux profile (peaked gradient, averaged over last 600 time units, $\rho_{*}=1 / 500$ ).

depend on an effective $\rho_{* \text { eff }}=\rho_{*} / \Delta_{T}$ (where $\Delta_{T}$ is related to $w_{T}$ of Eq. (3) or $\delta_{T}$ of Eq. (4), respectively). ${ }^{13,14}$

The weakening of interaction can be observed comparing Figs. 2 and 3 where the time-averaged heat fluxes are plotted as a function of the radial position for the peaked gradient profile for $\rho_{*}(x / a=0.5)=1 / 500$ and $\rho_{*}=1 / 150$. The turbulent flow is not affected by the presence of neoclassical effects for $\rho_{*}=1 / 500$ within the statistical error while it is significantly increased for $\rho_{*}=1 / 150$.

The time-averaged neoclassical heat flux $Q_{\mathrm{nc}}$ agrees well with the Chang-Hinton prediction ${ }^{15}$ in both cases if the neoclassical source is present. The discrepancy found in Fig. 3 for $x<0.15$ can be attributed to wide-orbit effects. ${ }^{16-18}$ If the neoclassical source is absent, non-zero though small transport (ca. 10\% of the physical NC transport) is measured in this channel. This reflects the fact that $Q_{\text {nc }}$ is the energy flux on the axisymmetric $\left(k_{y}=0\right)$ mode caused by the magnetic drift

$$
Q_{\mathrm{nc}}^{x}=\left\langle\int \frac{m v^{2}}{2}\left(\left[f_{1}\right]_{k_{y}=0} \boldsymbol{v}_{d}^{x}+F_{0}\left[\boldsymbol{v}_{\phi}^{x}\right]_{z}\right) \mathrm{d}^{3} v\right\rangle,
$$

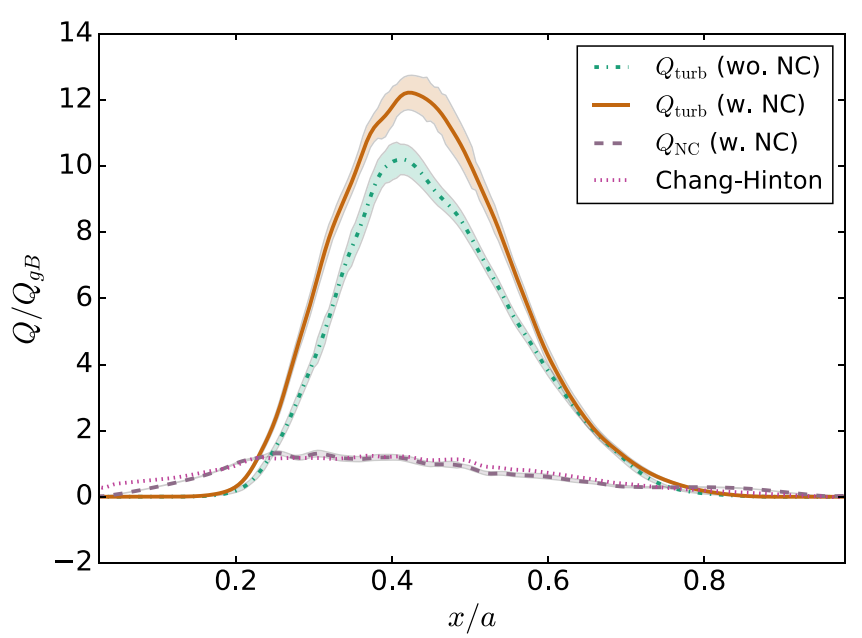

FIG. 3. Neoclassical and turbulent radial heat flux profile (peaked gradient, averaged over last 600 time units, $\rho_{*}=1 / 150$ ). whose dominant but not exclusive contribution is neoclassical effects. The second term is a small contribution from the radial component of the $\boldsymbol{E} \times \boldsymbol{B}$ drift caused by variations of the potential along $z$.

If we consider additional values for $\rho_{*}$ and compare the value of the time-averaged fluxes averaged over the radial region $x / a=0.4-0.6$ (with the maximal turbulent flow appearing at $x / a \approx 0.43$ for all cases), we arrive at the scaling shown in Fig. 4. The general trend that turbulent transport decreases with growing $\rho_{*}$ is a well established observation. ${ }^{14,19-22}$ Due to the narrow profile shape (see above), the convergence to the local limit occurs at relatively small $\rho_{*}$. At this point, it is important to remember that the fluxes are measured in units which imply the gyro-Bohm scaling, i.e., diffusive behaviour with the scale of the ion gyroradius and thus locality. Neoclassical heat transport outside of the near-axis region fulfils this condition and hence is mostly independent of $\rho_{*}$ in Fig. 4. The observed scaling of turbulent transport implies that system size effects start to play a role at large $\rho_{*}$. Below $\rho_{*}=1 / 300$ the scaling is quite close to Bohm-like, i.e., $Q / Q_{\mathrm{gB}} \propto 1 / \rho_{*}$. Comparing its values for a fixed system size, we find a systematic increase of $20 \%-30 \%$ for the system with neoclassical effects for $\rho_{*}>1 / 300$. It should be emphasized that this difference is not the neoclassical flux, which is measured separately and comes on top for an estimate of the total energy flux.

For $\rho_{*}=1 / 50$ both transport channels reach the same magnitude because the turbulent eddies' radial extent barely fits into the region where the temperature gradient can drive them. Since this case operates at the limits of the validity of the used gyrokinetic model and boundary artefacts can spread far across the radial domain, we do not analyse it beyond this qualitative observation.

The time resolved turbulent ion heat flux profiles in Fig. 5 both show the ripple structures which have been observed in several studies of ITG turbulence. ${ }^{14,23}$ Such structures of increased heat flux moving ballistically in- or outwards are commonly called avalanches and are features of so-called self-organized criticality. ${ }^{24-28}$ Reference 29

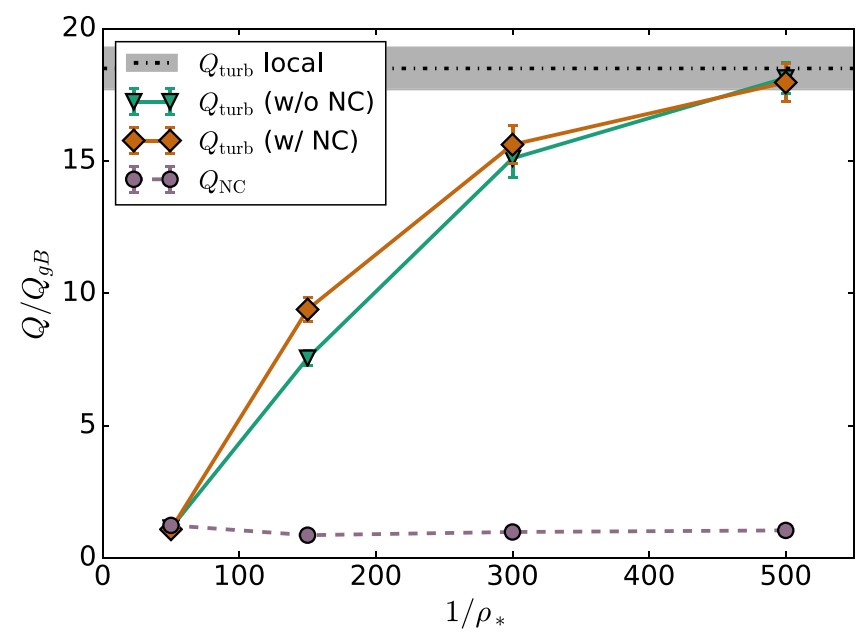

FIG. 4. Dependence on $\rho_{*}$ of the temporally and radially $(x / a=0.4-0.6)$ averaged radial turbulent and neoclassical heat fluxes in global ITG simulations. Dashed-dotted: flux-tube limit (shaded: uncertainty). 

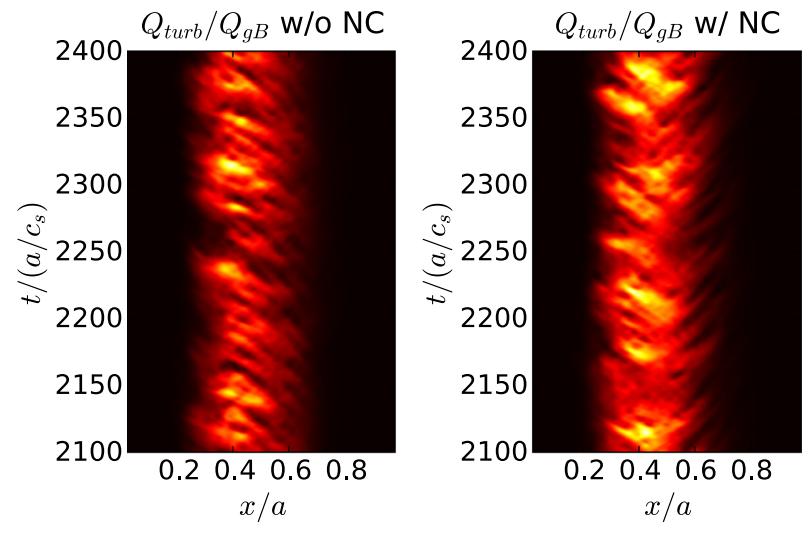

$0.0 \quad 2.5 \quad 5.0 \quad 7.5 \quad 10.012 .515 .017 .520 .022 .525 .0$

FIG. 5. Time resolved turbulent heat flux for $\rho_{*}=1 / 150$ (left: without, right: with $\mathrm{NC}$ source).

finds their direction to be connected with the sign of the $\boldsymbol{E} \times \boldsymbol{B}$ shear: $\omega_{\boldsymbol{E} \times \boldsymbol{B}}>0$ means outward movement, $\omega_{\boldsymbol{E} \times \boldsymbol{B}}$ $<0$ inward movement. Obviously, in Fig. 5 the presence of neoclassical effects changes their directions from a singular one (left) to a $\mathrm{V}$ shape (right). In consequence, we attribute the transport modification at least partly to a change in the zonal $\boldsymbol{E} \times \boldsymbol{B}$ shear pattern by the additional long range radial electric field which appears self-consistently when neoclassical effects are included in the Vlasov equation.

Indeed, if we look at the time averaged $\boldsymbol{E} \times \boldsymbol{B}$ shearing rate, $\omega_{\boldsymbol{E} \times \boldsymbol{B}}$, for $\rho_{*}=1 / 150$ (Fig. 6), the presence of neoclassical effects seems to align an area with low shear-the minimum of $E_{r}$-with the peak of the gradient at $x=0.5 a$. This is illustrated by the measured ITG growth rates $\gamma_{l o c}$ from local linear gyrokinetic simulations at the respective radial positions. Hence, the strongest turbulence drive coincides with the weakest damping rate. The reason for this large scale shape of the radial electric field lies in the radial force balance

$$
e E_{r}=-\nabla_{x} p+u_{t} B_{p}-u_{p} B_{t},
$$

which has contributions from the pressure gradient $\nabla p$ and the temperature gradient in the form of the neoclassical

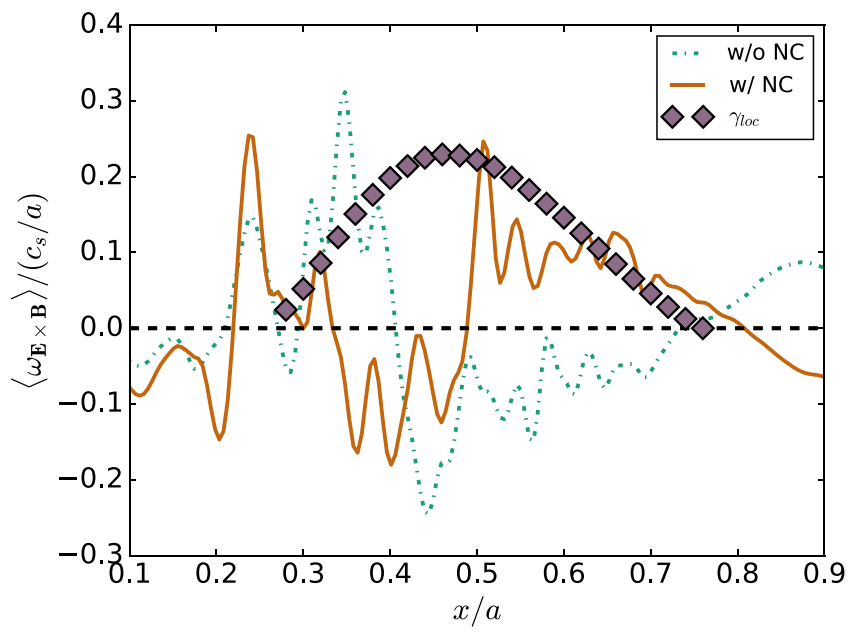

FIG. 6. Time averaged $\boldsymbol{E} \times \boldsymbol{B}$ shear in simulations without and with neoclassical effects, $\rho_{*}=1 / 150$, peaked gradient. Local linear ITG growth rate for comparison. poloidal velocity $u_{p} .{ }^{4}$ The toroidal velocity $u_{t}$ is a degree of freedom in the neoclassical theory. It can also be written in the following, normalised form:

$$
\left\langle u_{\| i} B\right\rangle=n_{i} T_{i}\left((k-1) \frac{\mathrm{d} \ln T_{i}}{\mathrm{~d} x}-\frac{\mathrm{d} \ln n_{i}}{\mathrm{~d} x}+\frac{E_{r}}{T_{i}}\right),
$$

where the poloidal rotation is now represented by the coefficient $k$. In Fig. 7, we compare the radial profile of this parameter from a simulation that includes the neoclassical source term with two predictions from the neoclassical theory, an approximate fit by Hinton and Hazeltine ${ }^{4}$ and the more precise derivation by Hirshman and Sigmar. ${ }^{30}$ It appears that the simulated radial electric field agrees well with the neoclassical transport theory but its turbulent contributions can locally also cause notable deviations from the Hirshman-Sigmar prediction.

In contrast to the differences in the spatial structure, root mean square radial averages of $\omega_{\boldsymbol{E} \times \boldsymbol{B}}$ yield similar values for simulations with and without neoclassical effects. This implies that the additional radial electric field does not generate a significant amount of absolute shearing rate (which would imply a decrease in turbulent transport).

In the case of smaller $\rho_{*}$, the spatial scales of zonal flows $\left(20-50 \rho_{i}\right)$ and long-range radial electric field (system size, i.e., $500 \rho_{i}$ ) are so far apart that the flow pattern is not generally affected: The direction of the avalanches can be found to change multiple times independently of the longrange background field.

\section{Profile shape}

A straightforward way to see the different contributions to the radial electric field and the statement made about the alignment of temperature gradient and $\boldsymbol{E} \times \boldsymbol{B}$ shear profile is to study systems with the flat-top profiles of Fig. 1(b). In this case, the pressure gradient cannot contribute to the $\boldsymbol{E} \times \boldsymbol{B}$ shearing rate in its flat-top region, so the neoclassical poloidal velocity plays a larger role.

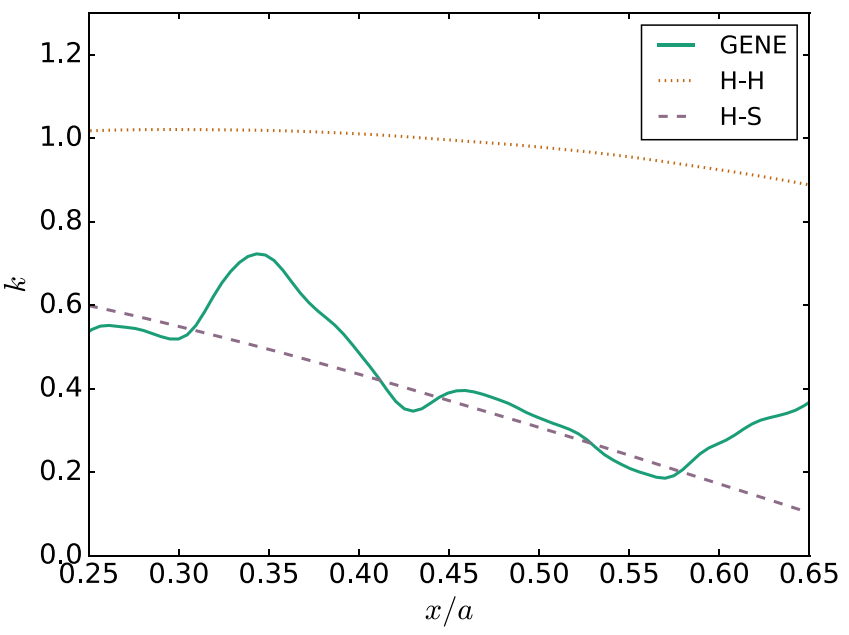

FIG. 7. Force balance parameter $k$ in a turbulent simulation with neoclassical effects $\left(\rho_{*}=1 / 150\right.$, peaked gradient) compared to neoclassical predictions from Ref. 4 (H-H) and Ref. $30(\mathrm{H}-\mathrm{S})$. 


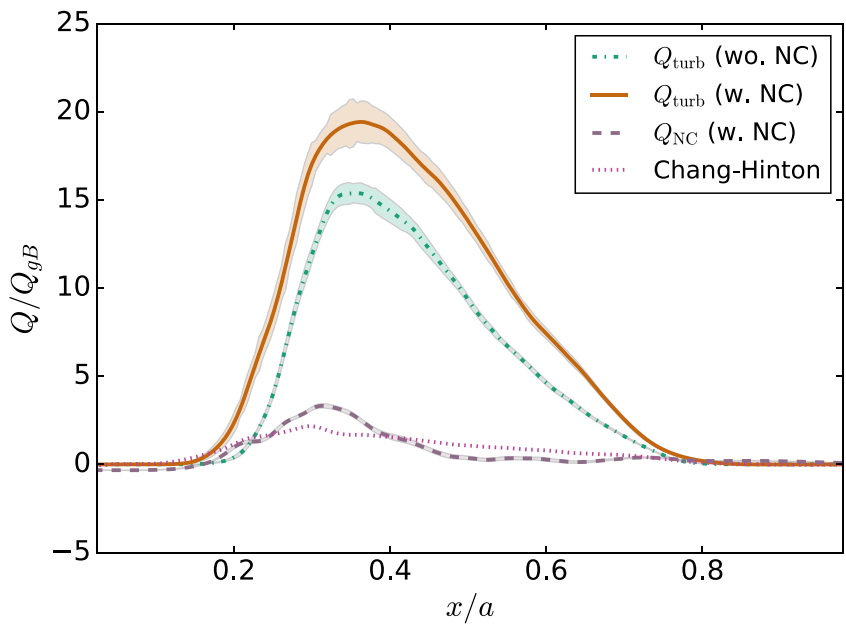

FIG. 8. Neoclassical and turbulent radial heat flux profile (flat-top gradient, averaged over last 600 time units, $\rho_{*}=1 / 150$ ).

We find again a consistently increased $(\sim 30 \%)$ turbulent ion heat flux in the simulation with $\rho_{*}=1 / 150$ when the neoclassical source is present (Fig. 8). When we examine the $\boldsymbol{E} \times \boldsymbol{B}$ shear profiles of the two cases in Fig. 9, the simulation with neoclassical source exhibits a visibly lower level of shear in the region $x / a \sim 0.5-0.8$. On the other hand, the shear is on average lower in the non-NC simulation around $x / a \sim 0.4$. In comparison with the local ITG growth rate, we find again that in the case with neoclassical effects a region with a low $\boldsymbol{E} \times \boldsymbol{B}$ shear coincides with high turbulent growth rates.

Consequently, for a flat-top gradient the alignment argument is insufficient when only considering the temperature and $\boldsymbol{E} \times \boldsymbol{B}$ shear to explain the turbulent flux modification by the neoclassical source. Although the gradient is the dominant quantity for determining the turbulence drive, it is not the only one: The magnetic shear $\hat{s}$, for example, also plays a role. $^{31,32}$ So the modification of the radial electric field by the presence of the neoclassical source in Eq. (1) remains a crucial effect.

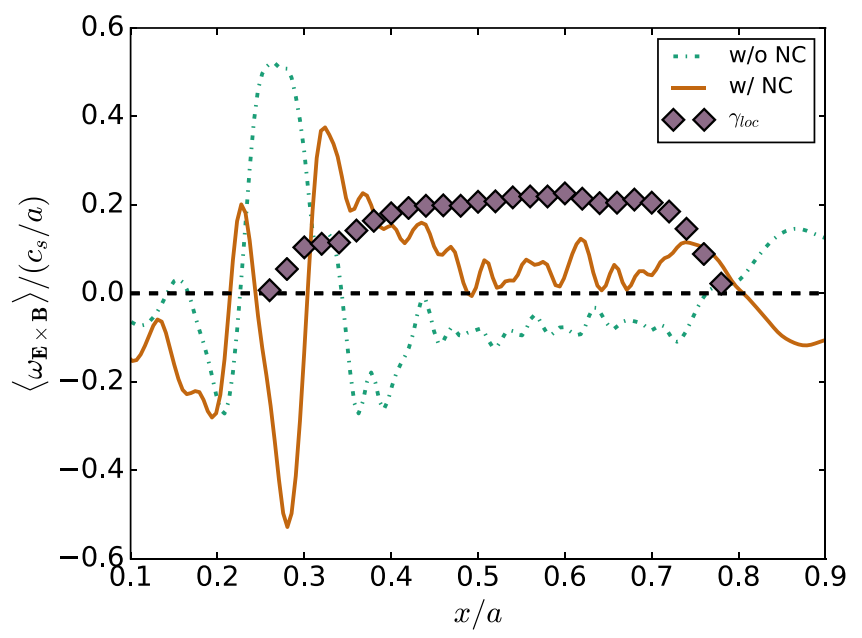

FIG. 9. Time averaged $\boldsymbol{E} \times \boldsymbol{B}$ shear in simulations without and with neoclassical effects, $\rho_{*}=1 / 150$, flat-top gradient. Local linear ITG growth rate for comparison.
The neoclassical flux in Fig. 8 now differs significantly from the Chang-Hinton prediction. Since the equivalent case for $\rho_{*}=1 / 500$ shows agreement at the same level as its peaked-profile counterpart in Fig. 2, this is another indication for an interaction between neoclassical and turbulent effects.

\section{Collisionality scaling}

The collision frequency in the results presented so far is chosen artificially high in order to achieve convergence of the neoclassical fluxes with a reasonable computational effort. The radial electric field is coupled to the parallel dynamics in the form of the radial force balance and establishes much faster. Hence, we investigate the influence of reducing the collision frequency which — as a side effectbrings it closer to a realistic value (as far as our model system can be considered realistic). This is achieved by setting the collisionality to $1 / 3$ or $1 / 10$ of its original value, i.e., $\nu_{*}(x=0.5 a)=0.095$ or $\nu_{*}(x=0.5 a)=0.029$. We will refer to these cases as medium and low collisionality, respectively.

The turbulent heat fluxes exhibit an interesting behaviour: Without the neoclassical source term (Fig. 10(a)), the energy flux is reduced with decreasing collision frequency as can be explained by a weaker collisional damping of zonal flows. $^{7,8}$ If neoclassical effects are present (Fig. 10(b)), however, this dependency vanishes and we find a flux profile which is very similar for all three collisionalities considered so far. Hence, the level of turbulent energy transport remains independent from the collisionality over an order of magnitude. A possible approach for an explanation is that the background radial electric field which is mostly independent of the collisionality in these cases provides a fixed structure to which the zonal flow pattern of the turbulence adapts. Further investigations, however, are needed to understand how this can counteract the collisional damping.

It should be noted that even if the independence of the fluxes holds numerically for $\nu \rightarrow 0$, it is not in contradiction to results comparing collisional and collisionless systems such as Ref. 6 because a different behaviour for the limit $\nu_{i} \rightarrow 0$ and $\nu_{i}=0$ is possible: A truly collisionless simulation needs to neglect the neoclassical source term, too. Otherwise, numerically necessary velocity space hyperdiffusion acts as a crude collision term and any observed neoclassical effects should be considered spurious.

The neoclassical radial heat fluxes (not plotted) behave qualitatively as expected in the form that they scale approximately linearly for smaller collisionality as they obey the standard neoclassical scaling in the banana regime. ${ }^{33}$

\section{EFFECTS OF NEOCLASSICAL PHYSICS IN FIXED-FLUX SIMULATIONS}

It is often argued that flux-driven simulations allow more self-organisation for the pressure profile and for transport. Thus, we now turn from gradient-driven to flux-driven scenarios. The heat source is no longer adaptive with the possibility of acting as a sink but a localized profile with fixed power input which is undeniably much closer to experimental conditions. This principle is the natural operation 


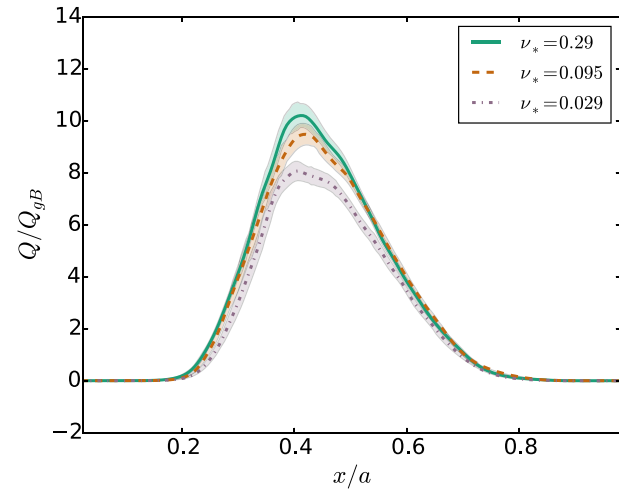

(a) without NC drive

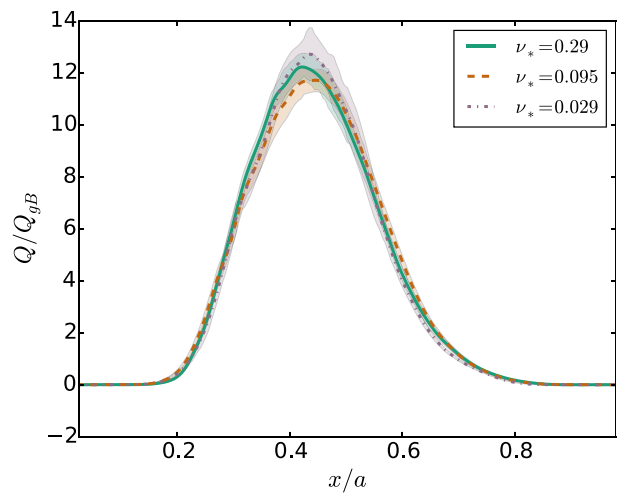

(b) with NC drive
FIG. 10. Collisionality scaling of the radial turbulent heat flux (values of $\nu_{*}$ at $x=0.5 a$ ). mode of full-f codes. Since this formalism automatically includes neoclassical effects, a number of results exist on the role they can play in turbulent flux-driven simulations. With the GYSELA code, ${ }^{34}$ for example, it is found that the poloidal rotation profile is dominated by the neoclassical mean flow and the $\boldsymbol{E} \times \boldsymbol{B}$ shearing rate attributed to it has a similar magnitude as the one caused by zonal flows. ${ }^{35}$ Temperature gradients also tend to establish closer to their critical values than in gradient-driven simulations so that the level of neoclassical heat transport can compete with its turbulent counterpart. ${ }^{36}$ In GT5D simulations, ${ }^{23,37}$ closeness to criticality is observed as well in the sense that a significant part of the turbulent heat flux occurs in the form of transient avalanches.

Since GENE is based on the $\delta f$ form of the gyrokinetic equations, a mechanism needs to be found in order to allow evolution of the temperature and density profiles while preserving $\delta f / f \ll 1$. This can be achieved by monitoring $\delta f$ and triggering a reset when its magnitude exceeds a predefined threshold: The current state of the total distribution function $F_{0}+\delta f$ is used to calculate new radial profiles which are then used as the initial state of a restarted simulation. This amounts to optimizing the initial condition of the gyrokinetic initial value problem until $\delta f$ remains within the assumptions on its magnitude.

The heat source in our simulations is an input of fixed shape equivalent to Eq. (4) (see also Ref. 36) positioned in the radial region $x / a=0-0.4$. Its amplitude is varied between simulations. The inner radial boundary of the simulation domain is floating (a Neumann boundary condition) thus allowing for temperature and density evolution due to the source. At the outer edge, temperature and density are fixed-formally a Dirichlet boundary condition-and a Krook type buffer zone is used to dissipate heat smoothly there: A term of the form $-k\left(x-x_{b}\right)^{2} f_{1}$ for $x>x_{b}$ is added to the gyrokinetic Vlasov equation, i.e., the outer boundary dampens the perturbed distribution function for $x>x_{b}$ $=0.89 a$ in our simulation. This sink model is similar to the one in Ref. 23 where the term has exponent 1.

The temperature and density profiles are initialized according to the flat-top gradient profiles of Eq. (4) with an amplitude significantly above the critical gradient for the temperature which accelerates their following evolution. While the density profile remains static due to the adiabatic electron approximation, the temperature evolves to adapt to the heat source and its evolution makes an adaptation of the background Maxwellian necessary. The threshold we use for this is $\left|f_{1} / F_{0}\right| \geq 0.18$. This is a relatively large value but triggering the adaption too often can lead to undesired numerical artefacts and we analyse simulations in a state when the last such reset is around 1000 time units past, i.e., the system has evolved to remain significantly below the threshold at that point. We study a system of $\rho_{*}=1 / 150$ for two different power input amplitudes $\left(\hat{S}_{0}=11250\right.$ and 45000 in units of $\left.n_{\mathrm{ref}} \rho_{*} c_{s} /\left(a v_{\mathrm{th}}^{3}\right)_{\mathrm{ref}}\right)$ ) and examine the result of including or neglecting neoclassical effects as well as the scaling with $\hat{S}_{0}$.

In Fig. 11, we present the time averaged temperature and heat flux profiles for the high strength source in the radial region $x / a=0.3-0.85$ where sources and sinks are not present or very weak. As can be seen in Fig. 11(a), the temperature gradients for the simulation with and without neoclassical source are nearly equal. The averaged turbulent heat fluxes in Fig. 11(b) accordingly have a similar magnitude. When the neoclassical source is present, however, some energy is also transported through the neoclassical flux channel. Hence, we observe a consistently lower turbulent energy flux in that case.

The time-resolved turbulent heat fluxes in Fig. 12 present a qualitatively different behaviour depending on the presence of the neoclassical source: Both cases exhibit the fishbone-like patterns familiar from Fig. 5. As pointed out in Section II B, the direction of these avalanches is related to the sign of the $\boldsymbol{E} \times \boldsymbol{B}$ shear. Sign changes of the shear can

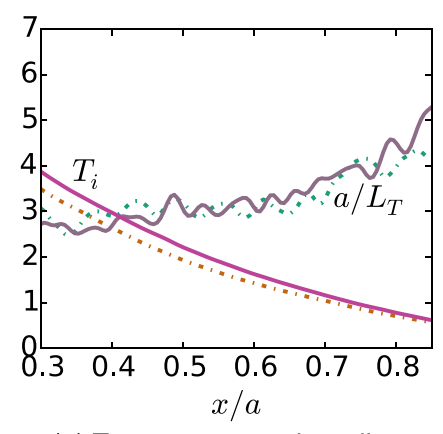

(a) Temperature and gradient

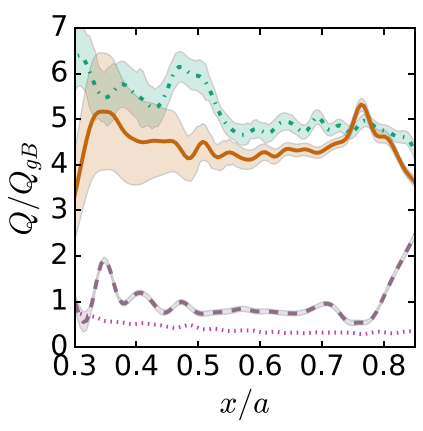

(b) Heat fluxes (dashed: NC, dotted: Chang-Hinton)
FIG. 11. Time averaged temperature profile and heat flux (averaged over last 1000 time units) in the source-free region for strong source amplitude $\left(\hat{S}_{0}=45000\right)$. Dashed-dotted: without, solid/dashed: with NC effects. 

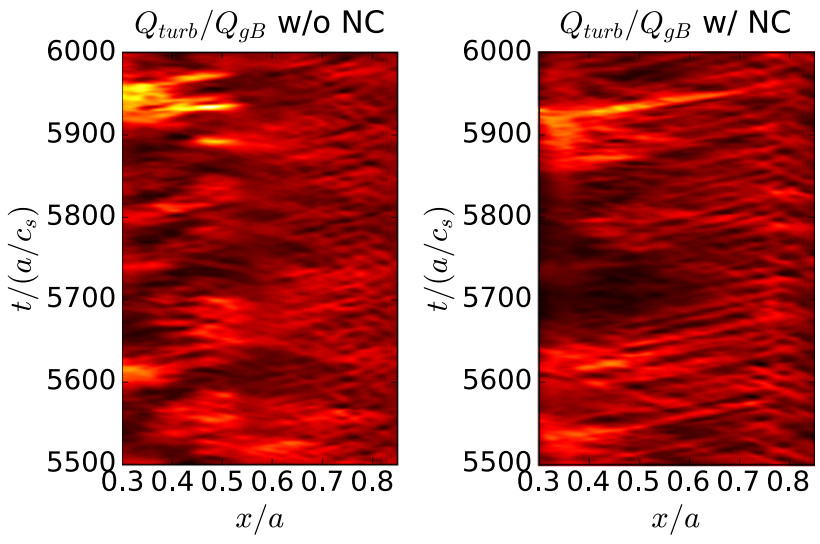

$\begin{array}{llllllllll}0.0 & 2.5 & 5.0 & 7.5 & 10.0 & 12.5 & 15.0 & 17.5 & 20.0 & 22.5\end{array}$

FIG. 12. Time resolved turbulent heat flux for strong source amplitude $\hat{S}_{0}=45000$ (left: without, right: with NC source).

lead to corrugations in the temperature profile in flux-driven simulations, and the resulting pattern has been given the name $\boldsymbol{E} \times \boldsymbol{B}$ staircase. ${ }^{38,39}$ Curiously, in our simulations, the only structure that fulfils the criteria for a stair step is occurring close to the sink region at $x \sim 0.75$ in Fig. 12. While an influence from the sink cannot be completely ruled out, the density gradient also changes significantly at this radial position (see Fig. 1(b)).

It is clear, however, when comparing the two simulations shown in Fig. 12 that just as in gradient-driven cases the avalanche pattern is fundamentally changed when neoclassical effects are included. Furthermore, there is a clear change to the intermittency of heat flux bursts: The system with neoclassical effects (Fig. 12, right) appears calmer with phases of low turbulent transport $(Q<5)$ lasting up to $100 \mathrm{a} / \mathrm{c}_{s}$. Without the neoclassical channel, on the other hand, these phases end already after $30-40 a / c_{s}$ (Fig. 12, left).

The explanation for this lies in the closeness of fluxdriven systems to criticality. ${ }^{23,36}$ When the temperature gradient decreases below the critical level, energy builds up and pushes it beyond criticality again. The collisional Dimits shift softening of the critical gradient smooths this behaviour slightly. Since the neoclassical transport channel has no critical gradient, its presence will slow down this mechanism: While it is in our case not sufficiently large to transport all input heat, it provides a leak to the energy build-up.

Closeness to criticality is expected to be stronger when we turn our attention to a system with halved heating power $\left(\hat{S}_{0}=11250\right)$ but otherwise identical parameters. As can be seen for the time-averaged quantities in Fig. 13(a), the system with neoclassical effects has a slightly higher ion temperature gradient which leads to higher turbulent flux in the outer region (Fig. 13(b)). Since we are comparing both systems for the same time window, the different convergence behaviour with and without neoclassical effects is probably responsible for this (neoclassical transport accelerates the evolution of the temperature profile towards its steady state). The region $x / a<0.5$ in Fig. 13(b), however, confirms the notion that part of the input power can be transported

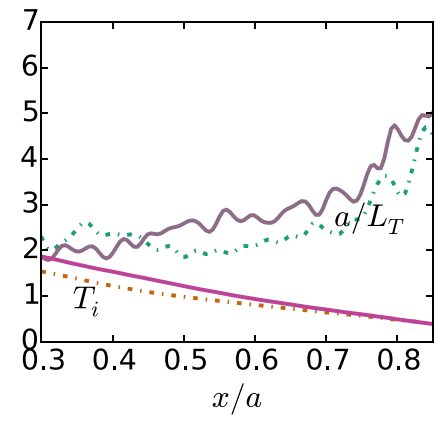

(a) Temperature and gradient

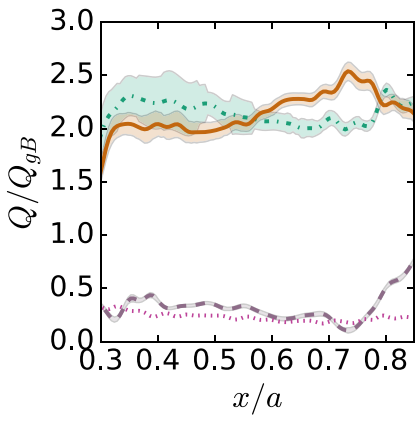

(b) Heat fluxes (dashed: NC, dotted: Chang-Hinton)
FIG. 13. Time averaged temperature profile and heat flux (averaged over last 1000 time units) in the source-free region for weak source amplitude $\left(\hat{S}_{0}=11250\right)$. Dashed-dotted: without, solid/dashed: with NC effects.

through the neoclassical channel and is hence missing in the turbulent flux.

For the time-resolved turbulent heat fluxes in Fig. 14, it is found that the presence of neoclassical transport reduces the strength of the intermittent turbulent bursts. In contrast to the case with strong source, however, the simulation without neoclassical effects (left) has a smaller burst frequency than its counterpart with neoclassical transport. The latter does not even exhibit a strong pattern of intermittency. It appears that the significant fraction of neoclassical transport inhibits the build-up of heat strong enough so that the system remains in a relatively steady state of weakly driven turbulence. This becomes more obvious when we compare the probability density functions of the turbulent heat fluxes in Fig. 15: In the simulation with neoclassical effects, the distribution lacks a tail end at high fluxes $\left(Q / Q_{g B}>4\right)$ and the quiet component $\left(Q / Q_{g B}<1\right)$ is significantly weaker.

Finally, when we collect the information from the two heating power scenarios and include a third intermediary heating amplitude, a comparison of the heat flux and gradients at the representative position $x / a=0.6$ in Fig. 16 demonstrates the well-established phenomenon of profile

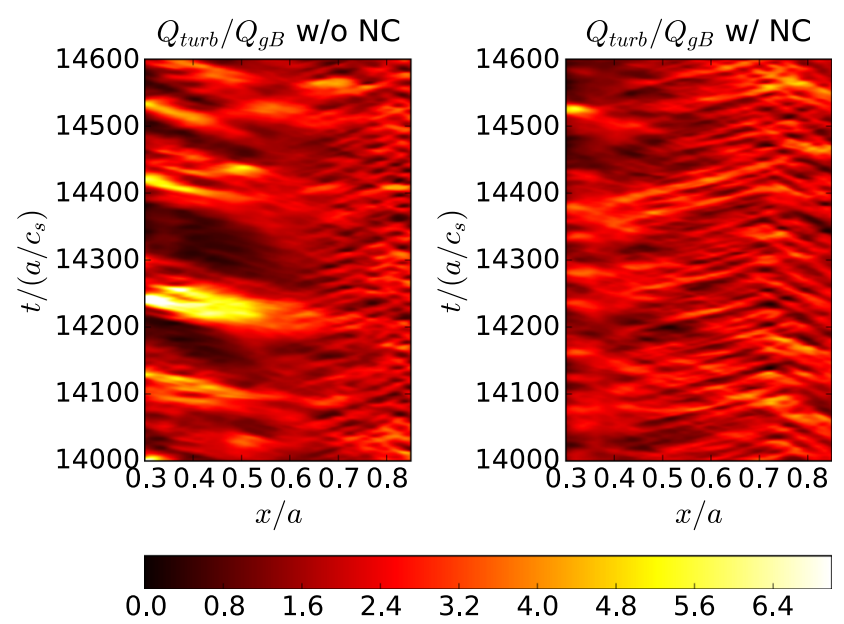

FIG. 14. Time resolved turbulent heat flux for weak source amplitude $\hat{S}_{0}=11250$ (left: without, right: with NC source). 


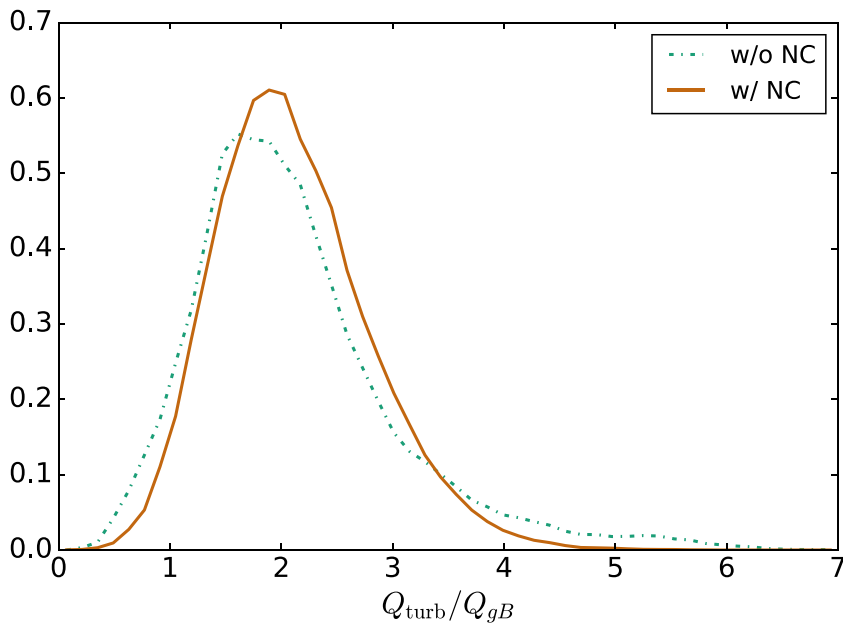

FIG. 15. Probability density function of the turbulent heat flux at $x / a=0.4$ -0.75 for weak source amplitude $\hat{S}_{0}=11250$.
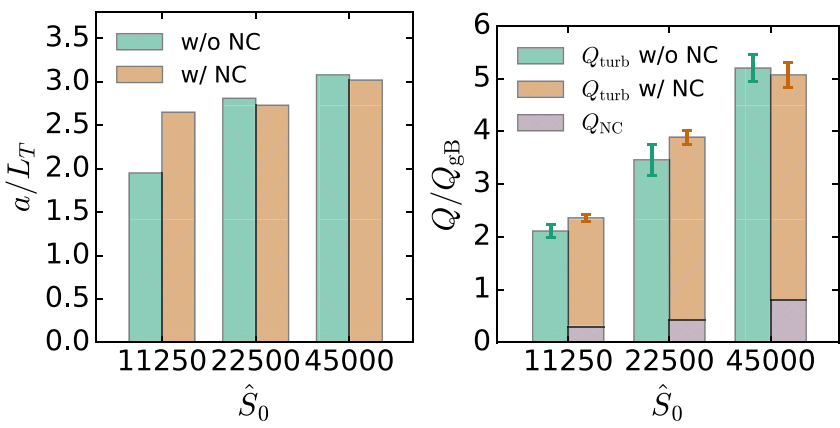

FIG. 16. Comparison of the time averaged temperature gradients and heat fluxes at $x / a=0.6$ for different power inputs.

stiffness ${ }^{40,41}$ in a very clear way: The temperature gradient remains nearly constant while the total heat flux at least doubles from the weakest to the strongest power input. This is the inverse situation compared to gradient-driven simulation where the fluxes are very sensitive to the input gradient. In addition, the sum of the neoclassical and turbulent heat flux for each power input agrees well with the turbulent heat flux from the corresponding non-neoclassical simulation.

\section{CONCLUSION}

In this article, radially global gyrokinetic simulations were used to explore the interaction between neoclassical and turbulent effects. In gradient-driven scenarios (i.e., with the time-averaged gradients fixed), it was demonstrated that this interaction only occurs when non-local effects play a role and that it vanishes for $\rho_{*} \leq 1 / 500$. This indicates that the analytical separation between the two effects in the local limit can be reproduced. It also implies that treating neoclassical and turbulent transport separately is valid for $\rho_{*} \lesssim 1 / 400$ where flux-tube simulations are justified by the turbulent scaling with $\rho_{*} \cdot{ }^{14,20}$ This is often assumed to be the case for modelling of experiments. For larger $\rho_{*}$ (or $\rho_{* \text { eff }}$ where the minor radius is replaced by the variation length of the gradient $^{13}$ ), however, including neoclassical effects in a turbulent simulation becomes strongly advisable. It was shown that the presence of neoclassical effects tends to reduce the dependence of turbulent transport on the collisionality. A possible explanation for this is that the additional radial electric field arising from the added neoclassical term determines the spatial positioning of the zonal flow pattern aligning zones with small $\boldsymbol{E} \times \boldsymbol{B}$ shear with ones of high turbulence drive. It was also possible to reproduce the phenomenon of radial heat flux avalanches whose direction follows the sign of the $\boldsymbol{E} \times \boldsymbol{B}$ shearing rate found in simulation results in the literature.

Finally, systems with fixed power input (flux-driven) were investigated where the temperature (gradient) profile evolves self-consistently according to a localized heat source. These simulations are considerably more complex and computationally intensive but allow more insight into self-organization phenomena. It was possible to reproduce the basic qualitative behaviour of results from full-f simulations such as the occurrence of intermittent bursts as a transport mechanism for energy because the system is closer to criticality. The frequency and amplitude of these bursts is modified when the additional neoclassical transport channel is present. By comparing different energy input powers, the experimentally and theoretically established phenomenon of profile stiffness was confirmed.

For future work, the flux-driven simulations can be analysed in more detail. Additionally, gradient-driven simulations can be performed with the self-consistent steady-state profiles in order to better understand the difference between the two approaches. For both approaches, it is also feasible to relieve some approximations made such as employing a more realistic model for the magnetic equilibrium or including electron dynamics in the investigation.

\section{ACKNOWLEDGMENTS}

The authors would like to thank J. Abiteboul and A. Bustos for helpful discussions on aspects of this work.

This work has been carried out within the framework of the EUROfusion Consortium and has received funding from the Euratom research and training programme 2014-2018 under Grant Agreement No. 633053. The views and opinions expressed herein do not necessarily reflect those of the European Commission. The research leading to these results has also received funding from the European Research Council under the European Union's Seventh Framework Programme (FP7/2007-2013)/ERC Grant Agreement No. 277870. The simulations in this work were carried out using the HELIOS supercomputer system at the Computational Simulation Centre of International Fusion Energy Research Centre (IFERC-CSC), Aomori, Japan, and the HYDRA supercomputer at the Max Planck Computing and Data Facility (MPCDF), Garching, Germany.

${ }^{1}$ L. Artsimovich, Nucl. Fusion 12, 215 (1972).

${ }^{2}$ S. C. Cowley, R. M. Kulsrud, and R. Sudan, Phys. Fluids B-Plasma 3, 2767 (1991).

${ }^{3}$ A. A. Galeev and R. Z. Sagdeev, Sov. J. Exp. Theor. Phys. 26, 233 (1968).

${ }^{4}$ F. L. Hinton and R. D. Hazeltine, Rev. Mod. Phys. 48, 239 (1976).

${ }^{5}$ R. C. Wolf, Plasma Phys. Controlled Fusion 45, 20-30 (2003). 
${ }^{6}$ T. Vernay, S. Brunner, L. Villard, B. F. McMillan, S. Jolliet, T. M. Tran, and A. Bottino, Phys. Plasmas 19, 042301 (2012).

${ }^{7}$ Z. Lin, T. S. Hahm, W. W. Lee, W. M. Tang, and P. H. Diamond, Phys. Rev. Lett. 83, 3645 (1999).

${ }^{8}$ P. H. Diamond, S.-I. Itoh, K. Itoh, and T. S. Hahm, Plasma Phys. Controlled Fusion 47, R35 (2005).

${ }^{9}$ F. Jenko, W. Dorland, M. Kotschenreuther, and B. N. Rogers, Phys. Plasmas 7, 1904 (2000).

${ }^{10}$ T. Görler, X. Lapillonne, S. Brunner, T. Dannert, F. Jenko, F. Merz, and D. Told, J. Comput. Phys. 230, 7053 (2011).

${ }^{11}$ X. Lapillonne, S. Brunner, T. Dannert, S. Jolliet, A. Marinoni, L. Villard, T. Gorler, F. Jenko, and F. Merz, Phys. Plasmas 16, 032308 (2009).

${ }^{12}$ A. M. Dimits, G. Bateman, M. A. Beer, B. I. Cohen, W. Dorland, G. W. Hammett, C. Kim, J. E. Kinsey, M. Kotschenreuther, A. H. Kritz, L. L. Lao, J. Mandrekas, W. M. Nevins, S. E. Parker, A. J. Redd, D. E. Shumaker, R. Sydora, and J. Weiland, Phys. Plasmas 7, 969 (2000).

${ }^{13}$ B. F. McMillan, X. Lapillonne, S. Brunner, L. Villard, S. Jolliet, A. Bottino, T. Görler, and F. Jenko, Phys. Rev. Lett. 105, 155001 (2010).

${ }^{14}$ T. Görler, X. Lapillonne, S. Brunner, T. Dannert, F. Jenko, S. K. Aghdam, P. Marcus, B. F. McMillan, F. Merz, O. Sauter, D. Told, and L. Villard, Phys. Plasmas 18, 056103 (2011).

${ }^{15}$ C. S. Chang and F. L. Hinton, Phys. Fluids 25, 1493 (1982).

${ }^{16}$ Z. Lin, W. M. Tang, and W. W. Lee, Phys. Rev. Lett. 78, 456 (1997).

${ }^{17}$ K. C. Shaing, R. D. Hazeltine, and M. C. Zarnstorff, Phys. Plasmas (1994present) 4, 771 (1997).

${ }^{18}$ P. Helander, Phys. Plasmas 7, 2878 (2000).

${ }^{19}$ Z. Lin, S. Ethier, T. S. Hahm, and W. M. Tang, Phys. Rev. Lett. 88, 195004 (2002).

${ }^{20}$ J. Candy, R. E. Waltz, and W. Dorland, Phys. Plasmas 11, L25 (2004).

${ }^{21}$ L. Villard, A. Bottino, S. Brunner, A. Casati, J. Chowdhury, T. Dannert, R. Ganesh, X. Garbet, T. Görler, V. Grandgirard, R. Hatzky, Y. Idomura, F. Jenko, S. Jolliet, S. K. Aghdam, X. Lapillonne, G. Latu, B. F. McMillan, F. Merz, Y. Sarazin, T. M. Tran, and T. Vernay, Plasma Phys. Controlled Fusion 52, 124038 (2010).

${ }^{22}$ Z. Lin, S. Ethier, T. S. Hahm, and W. M. Tang, Plasma Sci. Technol. 14, 1125 (2012).

${ }^{23}$ Y. Idomura, H. Urano, N. Aiba, and S. Tokuda, Nucl. Fusion 49, 065029 (2009).

${ }^{24}$ P. Bak, C. Tang, and K. Wiesenfeld, Phys. Rev. A 38, 364 (1988).
${ }^{25}$ D. E. Newman, B. A. Carreras, P. H. Diamond, and T. S. Hahm, Phys. Plasmas 3, 1858 (1996).

${ }^{26}$ B. A. Carreras, D. Newman, V. E. Lynch, and P. H. Diamond, Phys. Plasmas 3, 2903 (1996).

${ }^{27}$ J. Candy and R. E. Waltz, Phys. Rev. Lett. 91, 045001 (2003).

${ }^{28}$ B. F. McMillan, S. Jolliet, T. M. Tran, L. Villard, A. Bottino, and P. Angelino, Phys. Plasmas 16, 022310 (2009).

${ }^{29}$ B. F. McMillan, P. Hill, A. Bottino, S. Jolliet, T. Vernay, and L. Villard, Phys. Plasmas 18, 112503 (2011).

${ }^{30}$ S. Hirshman and D. Sigmar, Nucl. Fusion 21, 1079 (1981).

${ }^{31}$ J. Q. Dong, W. Horton, and J. Y. Kim, Phys. Fluids B-Plasma 4, 1867 (1992).

${ }^{32}$ F. Jenko, W. Dorland, and G. W. Hammett, Phys. Plasmas 8, 4096 (2001).

${ }^{33} \mathrm{P}$. Helander and D. Sigmar, Collisional Transport in Magnetized Plasmas, Cambridge Monographs on Plasma Physics (Cambridge University Press, 2002).

${ }^{34}$ V. Grandgirard, M. Brunetti, P. Bertrand, N. Besse, X. Garbet, P. Ghendrih, G. Manfredi, Y. Sarazin, O. Sauter, E. Sonnendrücker, J. Vaclavik, and L. Villard, J. Comput. Phys. 217, 395 (2006).

${ }^{35}$ G. Dif-Pradalier, V. Grandgirard, Y. Sarazin, X. Garbet, and P. Ghendrih, Phys. Rev. Lett. 103, 065002 (2009).

${ }^{36}$ Y. Sarazin, V. Grandgirard, J. Abiteboul, S. Allfrey, X. Garbet, P. Ghendrih, G. Latu, A. Strugarek, and G. Dif-Pradalier, Nucl. Fusion 50, 054004 (2010).

${ }^{37}$ S. Jolliet and Y. Idomura, Nucl. Fusion 52, 023026 (2012).

${ }^{38}$ G. Dif-Pradalier, P. H. Diamond, V. Grandgirard, Y. Sarazin, J. Abiteboul, X. Garbet, P. Ghendrih, A. Strugarek, S. Ku, and C. S. Chang, Phys. Rev. E 82, 025401 (2010).

${ }^{39}$ G. Dif-Pradalier, G. Hornung, P. Ghendrih, Y. Sarazin, F. Clairet, L. Vermare, P. Diamond, J. Abiteboul, T. Cartier-Michaud, C. Ehrlacher et al., Phys. Rev. Lett. 114, 085004 (2015).

${ }^{40}$ D. R. Baker, C. M. Greenfield, K. H. Burrell, J. C. DeBoo, E. J. Doyle, R. J. Groebner, T. C. Luce, C. C. Petty, B. W. Stallard, D. M. Thomas, M. R. Wade, and D.-D. Team, Phys. Plasmas 8, 4128 (2001).

${ }^{41}$ A. Peeters, O. Gruber, S. Günter, M. Kaufmann, H. Meister, G. Pereverzev, F. Ryter, A. Sips, J. Stober, W. Suttrop, G. Tardini, R. Wolf, H. Zohm, and the ASDEX Upgrade team, Nucl. Fusion 42, 1376 (2002). 